\title{
Non Machinable Volume Calculation Method for 5-Axis Roughing Based on Faceted Models through Closed Bounded Area Evaluation
}

\author{
Gandjar Kiswanto ${ }^{1}$, Himawan Hadi Sutrisno ${ }^{2}$ and Jos Istiyanto ${ }^{1}$ \\ ${ }^{1}$ Dept. of Mechanical Engineering, Universitas Indonesia, Depok, Indonesia \\ ${ }^{2}$ Mechanical Engineering, Universitas Negeri Jakarta (State University of Jakarta), Indonesia
}

\begin{abstract}
Abtract. The increase in the volume of rough machining on the CBV area is one of the indicators of increased efficiencyof machining process. Normally, this area is not subject to the rough machining process, so that the volume of the rest of the material is still big. With the addition of CC point and tool orientation to CBV area on a complex surface, the finishing will be faster because the volume of the excess material on this process will be reduced. This paper presents a method for volume calculation of the parts which do not allow further occurrence of the machining process, particulary for rough machining on a complex object. By comparing the total volume of raw materials and machining area volume, the volume of residual material,on which machining process cannot be done,can be determined. The volume of the total machining area has been taken into account for machiningof the $\mathrm{CBV}$ and non $\mathrm{CBV}$ areas. By using delaunay triangulation for the triangle which includes the machining and CBV areas. The volume will be calculated using Divergence(Gaussian) theorem by focusing on the direction of the normal vector on each triangle. This method can be used as an alternative to selecting tothe rough machining methods which select minimum value of nonmachinable volume so that effectiveness can be achieved in the machining process.
\end{abstract}

\section{Introduction}

In the manufacturing industry, especially for manufacturers of dies and molds, efficiency in the process of machining becomesan issue for the machining cost reduction. The use of the 5 -axis milling machine provides an opportunity for the single step processto form a product. In general, machining processare divided into 3 groups such as roughing, semi finishing and finishing. The rough machining process has biggerexcess materials if compared to the following two machining methods, although the results achievedhas a high level of roughness.

Until now, the development of the rough machining methods continues to be developed by several researchers. Some of them use faceted model as the database $[1,2]$, but some use parametric model as the basis of calculation $[3,4]$.

In fact, forms of the models that will be created using the process of machining extremely vary. This allows the process to continue even in certain forms containing nonmachinable sections; in other words, there is an obstacle for the tool to enter the area $[5,6]$. This part is commonly referred toas the close bounded volume (CBV area), where the methodfor detecting and determining the position of the $\mathrm{CBV}$ area on a workpiece model is as introduced by G. Kiswanto et al [6]. CBV area, generally a signal that in such area there is a hitch so that it requiresa special strategy such as the addition of $\mathrm{CC}$ point to the area [7]. However, the existence of evasion of damage due to over cuttingin the rough machining process is also important [8].

For the purpose of calculating the machiningvolume, the extract volume method for each position of the tool has been introduced by C. Park [9]. On the basis of the final part as the base of comparison, a part that is supposed to be disposed of is calculated using 3D booleans.Meanwhile ,the calculation of the effective area for rough machining process has also ever been introducedby Zhiwei Lin et al [10]. This method uses the slicing plane to get the particular Z-axis value as a layer in the rough machining process.

As the basis for calculation, the facet model used to obtain the total volume of material will be described in the procedure below.

\section{Development of methods}

From the literature study, the feature of a workpiece model and machining area of a final form of a product can be determined using several methods. [11]. Slicing plane method is used to identify primitive features in one model. In the development of the methods for calculating the volume of the remnant of the materials in this study, the slicing method is also usedto identify the final part, $\mathrm{CBV}$ and non $\mathrm{CBV}$ areas as described in Figure 1 below. 


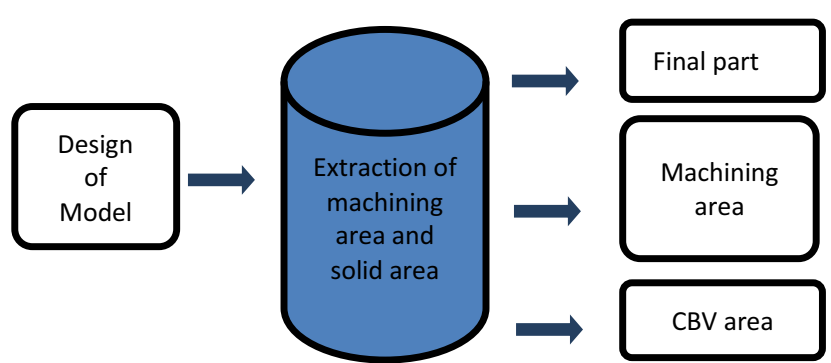

Figure 1. Extract of machining area and solid area

Facet model is used as the basis for the data obtained from the CAD system with STL files format. The volume of the area in which machining process cannot take place is the difference between the raw material and total machining area, as described in Figure 2 below.
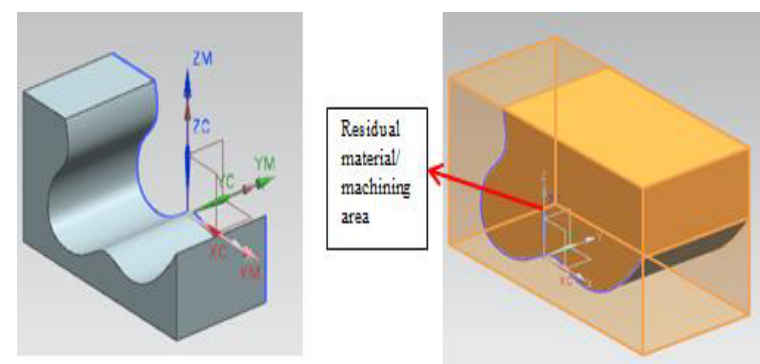

2a. Final part 2b. residual stock.

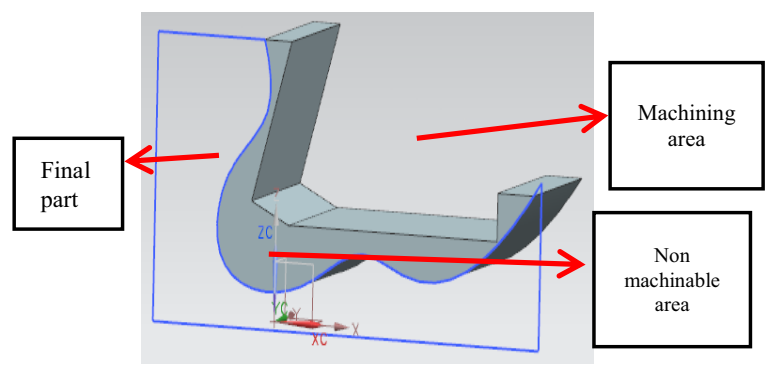

Figure 2. Separation of machining residual area.

\subsection{Determining total volume of raw material}

To begin with identification of the dimension of the object, in a 3 dimensional place, the location of the furthest point is identified as maximum valuesof $X$ (X_max), $Y\left(Y \_m a x\right)$ and $Z\left(Z_{-}\right.$max) while the nearest to the axis is the minimuum values of $\mathrm{X}$ (X_min), $\mathrm{Y}\left(\mathrm{Y} \_\right.$min) and $Z\left(Z_{-}\right.$min). These points are determined from the triangle that forms models of STL files. The total volume of raw materialis as follows:

$$
\begin{aligned}
& \text { raw material volume }=\text { length of part in } X \text { - } \\
& \text { axisdirection * length ofpart in Y-axisdirection * } \\
& \text { length of part in Z-axisdirection } \\
& =\left(\max \_x-\min \_x\right) *(\max y \text {-min_y }) *\left(\max _{-} z-\right. \\
& \text { min_z }
\end{aligned}
$$

As for the final part volume of a model with the basis of data in the form of faceted model using Gauss/Divergence theorem as described below:

$$
\text { Final part volume }=\iint_{G} \underset{n}{\rightarrow} d s=\iiint d i v \underset{G}{\rightarrow} d V
$$

The divergence theorem states that the outward flux of a vector field through a closed surface is equal to the volume integral of the divergence over the region inside the surface.

\subsection{Determining machining volume in CBV area}

To determine Close Bounded Volume (CBV area), grouping the point cloud in the machining area is doneby CBV evaluation concept[6] with the end result of this concept is $\mathrm{CBV}$ area and non $\mathrm{CBV}$ area or Open BoundedVolume (OBV area). This concept based on point cloud evaluation with slicing line method. Point cloud formation on all parts of the raw material, based on the point cloud formation in the XY plane by the distance between the point cloud is determined by the value of densityseen in figure $3 \mathrm{a}$. Where previously the point cloud formed on XY plane base was also formed in each $X Y$ place with a certain $Z$ value. In figure $3 b$, the entire point cloud that is formed (at each level of $Z$-axis ) will later be used as a $\mathrm{CC}$ point for machining process.

By adopting the slicing line with fast, minimum storage ray/triangle intersection method [12] which is applied for every point cloud along $\mathrm{XY}$ plane on $\mathrm{Z}=0$ (psudocode below), intersection of lines on the workpiece surface tiangle is used as the basis for determining the CC point for machining process seen in Figure 3c. The point cloud defined as $\mathrm{CC}$ point is the one outside two intersections from the slicing line in the vertical slicing line that covers workpiece,

for $\mathrm{i}=1$ :size(points_cloud, 1 )

for $\mathrm{j}=1$ :size(points_cloud, 2 )

$\mathrm{x}=$ points_cloud $(\mathrm{i}, \mathrm{j}, 1)$;

$\mathrm{y}=$ points_cloud $(\mathrm{i}, \mathrm{j}, 2)$;

$\mathrm{z}=$ points_cloud $(\mathrm{i}, \mathrm{j}, 3)$;

z_max $=$ max_min $(1,3)$;

$\operatorname{line}\left([\mathrm{x} ; \mathrm{x}],[\mathrm{y} ; \mathrm{y}],\left[\mathrm{z} ; \mathrm{z} \_\max \right]\right.$,

'Color','b','LineWidth',1,'LineStyle','-');

end

end

CBV area is area between two solid models in one slicing line as Figure $3 \mathrm{~d}$ below. Data of the slicing line on the previous step is used to classify CC point on each surface machining layer (based on intersections of slicing line with triangle surface).

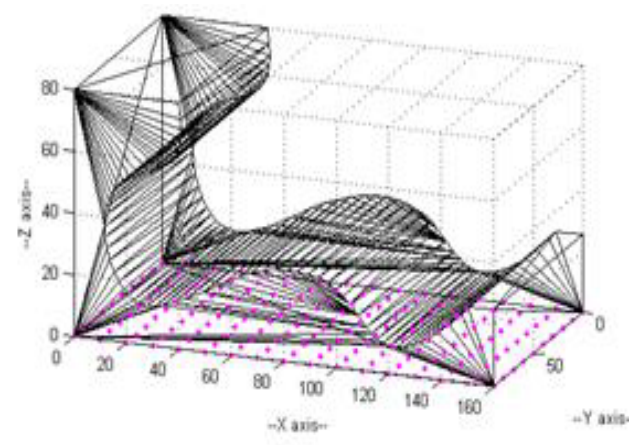

3a. The formation of point cloud in the XY plane 


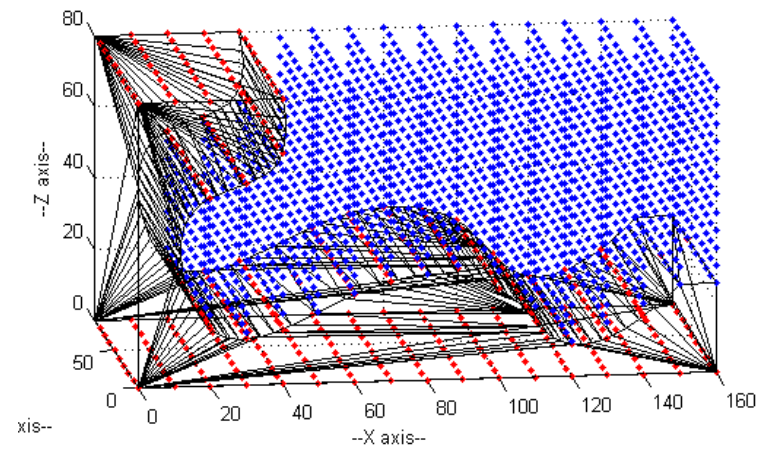

3b. The formation point cloud in the machining area

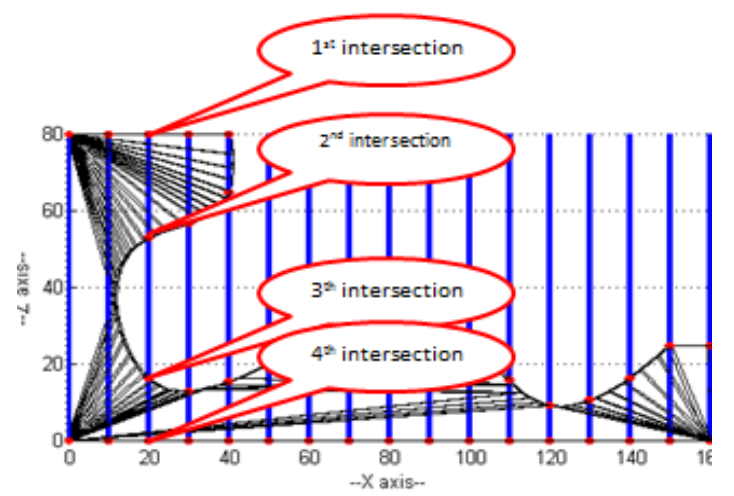

3c. licing line of every point cloud

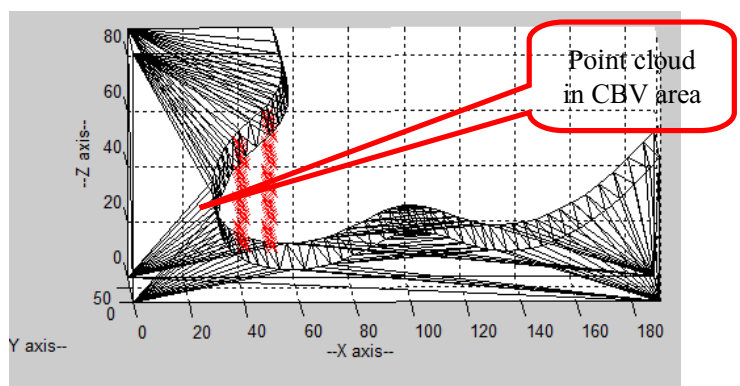

3d. Point cloud in CBV area

Figure 3. Formation of point cloud and Slicing line of every point cloud

Then, the position of the point cloud inside the final part, $\mathrm{CBV}$ area andoutside the $\mathrm{CBV}$ area can be identified[7]. Then, the machining volume in $\mathrm{CBV}$ area depends on a dimension of tools as used and vector direction of $\mathrm{CC}$ point in the machining of $\mathrm{CBV}$ area. In every direction of machining ( $\mathrm{u}$ and $\mathrm{v}$ direction) the volumeof each CC point will be summed up as shown in Figure 4 below. Whereas, the volume for CBV machining area equalsto feeding tools track from the nearest $\mathrm{CC}$ point outside $\mathrm{CBV}$ until $\mathrm{CC}$ point inside $\mathrm{CBV}$ with the inclination angle of $\Theta$ and the track is in the form of a cicular section. Therefore, the result of the calculation will be equal to thevolume of the circular section plus the semi-cylindrical volume (tool diameter) seen in Figure 5. Algorithm: cilindrical volume calculation

Input: angle degree norm vektor, tool diameter

Output: CBV part volume

Define $\mathrm{u}$ or $\mathrm{v}$ formed the rotationangle between $\mathrm{u}$ and $\mathrm{v}=$ $\operatorname{atan} 2(\operatorname{norm}(\operatorname{cross}(\mathrm{u}, \mathrm{v})), \operatorname{dotn}(\mathrm{u}, \mathrm{v}))$

cbv_part_volume $=$ angle $/ 2 \pi *$ area $*$ heigth intersected_cbv_volume $=$ angle / $(2 *$ pi $) *($ pi $*$ tool_length - vertical_stepover) $\wedge$ 2) * $\left(\max \_\min (1,2)-\max \_\min (2,2)\right)$

cbv_part_volume $=\quad \overline{c b v \_p a r t \_v o l u m e}$ intersected_cbv_volume

total_cutting_volume $=$ total_cutting_cbv_volume + obv_part_volume;

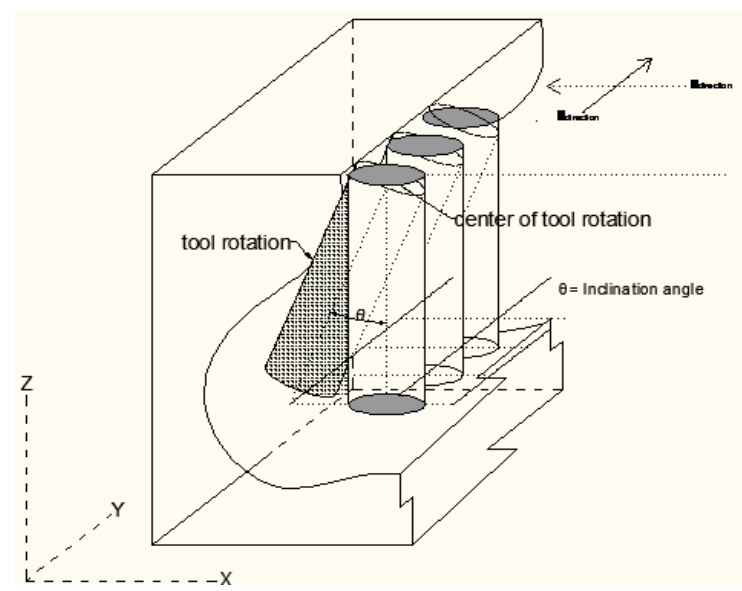

Figure 4. Machining volume in CBV area.

Figure 5. Cilindrical volume

\subsection{Determining machining volume in non CBV area}

In determining the volume of machining on a non $\mathrm{CBV}$ area(commonly called as OBV area), the volume of consumption will depend on number of $\mathrm{CC}$ point at each layer (Z-level) and diameter of the toolsis used. Due to the direction of orientation on OBV this area with z-axis normal then, volume for eachfour $\mathrm{CC}$ point will equal to the volume of a boxwith a length and width equal with density also a height equal with dept of cut.

Totalmachiningvolume in $O B V$ area $=\Sigma$ cube(every four CCpoint *dept of cut or layering)

\subsection{Volume of non machinable area}

Based on the whole calculation of raw material, the final part and total volume of the machining area (CBV area plus OBV area), the calculation of non machinable volume can be calculated as the total volume of raw material less the volume of the final part along with the 
machining area. This can be expressed mathematically as follows:
Non machinable volume = raw material volume (part volume + volume of machining area)

Table 1. Implementation method

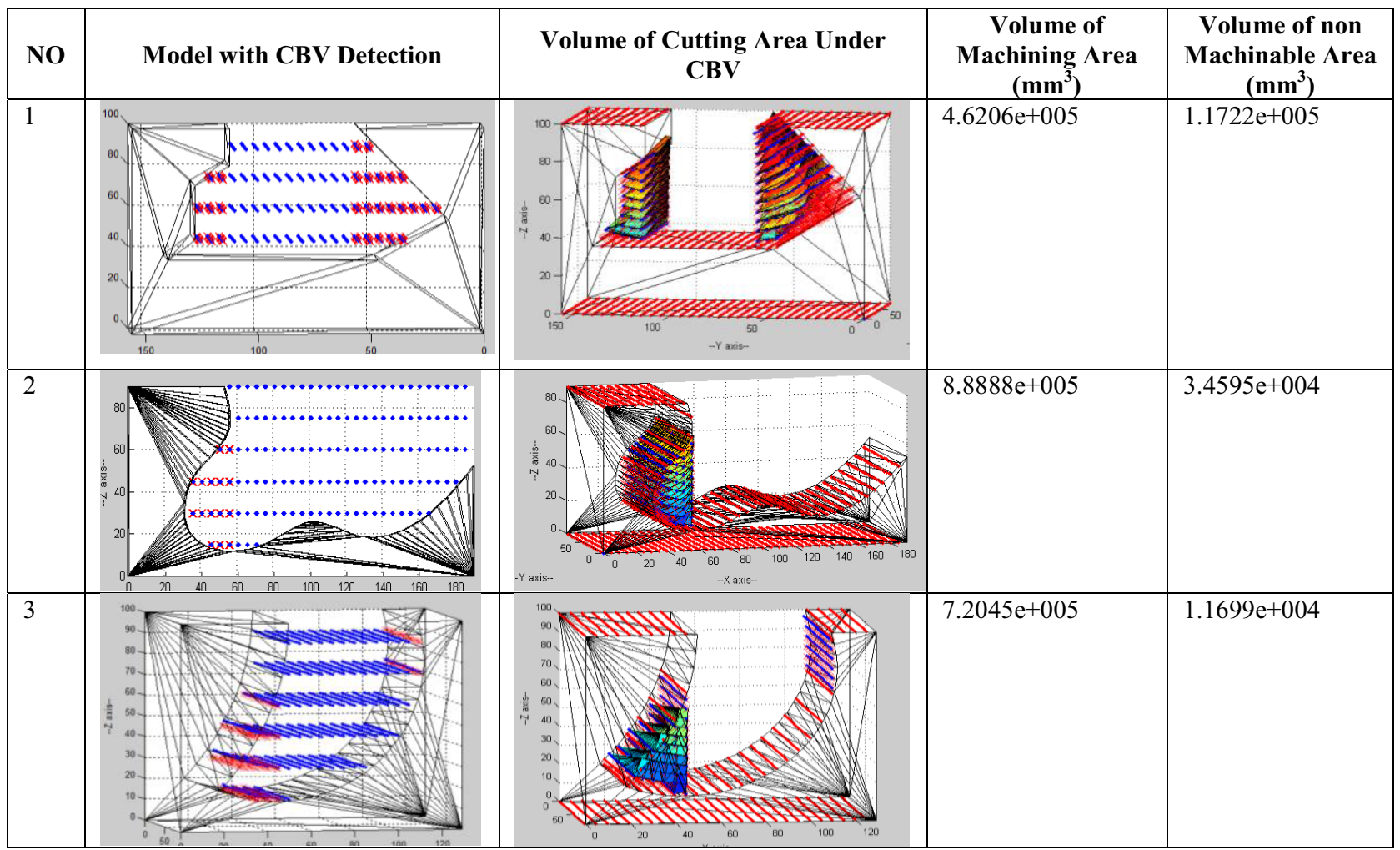

\section{Implementation}

In order to justify whether or not this method can be used for each model of the workpiece, particularly forcomplex forms containing CBV area, this method is tested on the forms of models below.

\section{Conclusion}

The results of simulation of some of workpieces and models show that the volume of any non machinable area can be exactly known; therefore, by utilizing this method can be used in general to select 5-axis rough machining methods that produce the smallest non-machinable. The less residual material left for 5-axis roughing, the finishing process will be faster so the machining time becomes more efficient.

\section{References}

1. M. Balasubramaniam, P. Laxmiprasad, S. Sarma, and Z. Shaikh, "Generating 5-axis NC roughing path directly from a tesselated representation," ComputerAided Design, vol. 32, p. 17, (2000)

2. B. L. G. K. J. P. Kruth, "Development of a Five axis Milling Tool Path Generation Algorithm based on faceted model," (2003)

3. H.-Z. Fan, W. Wang, and G. Xi, "A novel five-axis rough machining method for efficient manufacturing of centrifugal impeller with free-form blades," The
International Journal of Advanced Manufacturing Technology, vol. 68, pp. 1219-1229, (2013)

4. R. Qi, W. Liu, H. Bian, and L. Li, "Five-axis rough machining for impellers," Frontiers of Mechanical Engineering in China, vol. 4, pp. 71-76, (2009)

5. K. Suthunyatanakit, E. L. J. Bohez, and K. Annanon, "A new global accessibility algorithm for a polyhedral model with convex polygonal facets," Computer-Aided Design, vol. 41, p. 14, (2009)

6. G. Kiswanto. and A. Y. T. Panuju, "Development of closed bounded volume (CBV) grouping method of complek faceted model through CBV Boundaries identification.," IEEE, vol. 3, p. 5, (September 2010)

7. G. Kiswanto, H. H. Sutrisno, and J. Istiyanto, "Development of Initial Tool Orientation Method At Close Bounded Area for 5-Axis Roughing Based On Faceted Models," ICMM, vol. 3, (2017)

8. G. Kiswanto, B. Lauwers, and J.-P. Kruth, "Gouging elimination through tool lifting in tool path generation for five-axis milling based on faceted models," Int. J Adv Manuf Technol, vol. 32, p. 21, (2007)

9. M. Chang and S. C. Park, "An algorithm to extract machining volumes," Int. J Adv Manuf Technol, vol. 36, p. 8, 5 (January 2007)

10. Z. Lin, J. Fu, H. Shen, and W. Gan, "Efficient cutting area detection in roughing process for meshed surfaces," The International Journal of Advanced Manufacturing Technology, vol. 69, pp. 525-530, (2013) 
11. G. Kiswanto and M. Azka, "Automatic Part Primitive Feature Identification Based on Faceted Models," international journal of computer science issues, vol. 9, p. 7, (2012)
12. T. Moller and B. Trumbore, "Fast MinimumStorage RayTriangle Intersection," (1997) 